\title{
Occupational biological risk knowledge and perception: results from a large survey in Rome, Italy
}

\author{
Maria De Giusti ${ }^{(a)}$, Carmela RN Corrao(a), Alice Mannocci( $^{(a)}$, Caterina Palazzo ${ }^{(a)}$, \\ Roberta Riccardi $^{(a)}$, Silvia Lisa Schmidt( ${ }^{(a, b)}$, Sabrina Sernia ${ }^{(a, c)}$ and Giuseppe La Torre ${ }^{(a)}$ \\ ${ }^{(a)}$ Dipartimento di Sanità Pubblica e Malattie Infettive, Sezione di Igiene, \\ Sapienza Università di Roma, Rome, Italy \\ (b) Amsterdam University College, Amsterdam \\ (c) Centro di Medicina Ocupazionale, Sapienza Università di Roma, Rome Italy
}

\begin{abstract}
Background. A cross-sectional survey on knowledge and perception of occupational biological risk among workers in several occupations was carried out in the industrial area of Rome. Methods. The study was carried out in the period of March-April 2010 using a questionnaire with 33 items on the following areas: a) socio-demographic data; b) perception of the biological risks in ordinary occupational activity; c) knowledge about biological risks; d) biological risks in the working environment. The questionnaire was submitted to a convenience sample of workers of an industrial area in Southern Rome. Results. 729 participants entered the study from the following work activities: food, catering, service, farming and breeding, healthcare, school and research (males 57.2\%; mean age 37.4 years, $\mathrm{SD}=10.9$ ). Significant associations were found between different activity areas with respect to the relevance of the biological risk ( $\mathrm{p}$ $=0.044)$ and the perception of the biological risk $(\mathrm{p}<0.001)$. With respect to vehicles of infectious agents, the highest percentages of the most common biological risk exposures were: air and physical contact for the catering and food group, $66.7 \%$ and $61.90 \%$ respectively; air and blood for the health and research group, with $73.50 \%$ and $57.00 \%$ respectively; and physical contact and blood for the service group, 63.10 $\%$ and $48.30 \%$. Significant difference of proportions were found about the prevalent effect caused by the biological agents was the occurrence of infectious diseases (59.90\% food group, $91.60 \%$ health and research and $79.30 \%$ service group) $(\mathrm{p}<0.001$ ). The perception of knowledge resulted in a good rank (sufficient, many or complete) in the food and catering group, $78.3 \%$ with significant difference compared to other professions $(\mathrm{p}<0.001)$. Conclusions. All participants show good knowledge the effects induced by biological agents and it is significant that almost half of the respondents are aware of the risks concerning allergies. Nevertheless, it is surprising that this risk is known mainly by workers of service, farming and breeding, to a greater extent than it is known by health workers.
\end{abstract}

Key words: biological risk, knowledge, perception, workers.

Riassunto (Conoscenza e percezione del rischio biologico occupazionale: risultati di una indagine condotta a Roma, Italia). Introduzione. È stato condotto uno studio trasversale nell'area industriale di Roma relativo alle conoscenze e alla percezione del rischio biologico occupazionale fra lavoratori di diverse tipologie. Metodi. Lo studio è stato condotto nel periodo marzo-aprile 2010 utilizzando un questionario che indagava sulle seguenti aree di interesse: a) dati socio-demografici; b) percezione del rischio biologico nelle attività lavorative ordinarie; c) conoscenza dei rischi biologici; d) rischi biologici nell'ambiente lavorativo. Risultati. Hanno preso parte allo studio 729 partecipanti delle seguenti categorie: alimentazione, catering, servizi, agricoltura e allevamento, sanità, scuola e ricerca (maschi 57,2\%; età media 37,4 anni, SD = $10,9)$. Sono state riscontrate associazioni significative fra le differenti tipologie lavorative e la rilevanza $(p=$ $0,044)$ e la percezione $(p<0,001)$ del rischio biologico. In relazione al tipo di veicolo di infezione, le percentuali più elevate di rischio biologico riportate dai rispondenti riguardano: 1'aria $(66,7 \%)$ e il contatto fisico $(61,9 \%)$ per il gruppo dei lavoratori del settore alimentare e catering; l'aria $(73,5 \%)$ e il sangue $(57,0 \%)$ per il gruppo dei professionisti sanitari e dei ricercatori; contatto fisico $(63,1 \%)$ e sangue $(48,3 \%)$ per il gruppo dei lavoratori dei servizi. Sono state riscontrate differenze significative nelle proporzioni relative all'effetto prevalente causato da agenti biologici (59,9\% nel gruppo dei lavoratori degli alimenti, 91,6\% nel gruppo dei professionisti sanitari e dei ricercatori e 79,3\% nel gruppo dei servizi) $(\mathrm{p}<0,001)$. Conclusioni. Tutti i partecipanti mostrano una buona conoscenza degli effetti indotti dagli agenti biologici ed è significativo che circa la metà di essi sia consapevole dei rischi relative alle allergie. È tuttavia sorprendente che questo rischio sia conosciuto meno dai professionisti sanitari rispetto alle altre categorie lavorative considerate.

Parole chiave: rischio biologico, conoscenze, percezione, lavoratori. 


\section{INTRODUCTION}

Occupational transmission of infectious diseases is responsible for an estimated 320000 deaths/year worldwide and 5000 deaths/year in the European Union (EU) [1]. Occupational exposures to biological agents can occur in several sectors, such as health, agriculture, forestry, animal husbandry, food, veterinary, biotechnology, waste processing and disposal, laboratories, and dentistry, furniture factories, biomass production, cigarette and cigar manufacture, military and penitentiary personnel, drivers, office workers and waste management [2-17].

According to the World Health Organization (WHO), each year many healthcare workers in Europe are at risk of acquiring an infectious disease after a percutaneous exposure; 304000 individuals are exposed to Hepatitis B virus (HBV); 129000 are exposed to Hepatitis $\mathrm{C}$ virus (HCV), and 22000 are exposed to Human Immunodeficiency virus (HIV). The likelihood of acquiring an infection after occupational exposure is $<0.3 \%$ for $\mathrm{HIV}, 0.5 \%$ for $\mathrm{HCV}$, and $18 \%-30 \%$ for HBV [18]. Although there is evidence that knowledge among healthcare personnel about preventive measures in occupational exposure to blood and body fluids is adequate, transferring this knowledge into practice remains inadequate [19].

Data on workers' attitudes and risk perception about biological risk are poor. Several comments declare detecting "the perception" of biological risk, but actually detect only the knowledge about risk. There is evidence that failure to report exposure to biological agents is based on perception of a non significant risk $[20,21]$, and that perception of high risk is particularly related to involuntary exposures against risk factors that are neither seen nor smelled, especially when they involve serious diseases, such as AIDS [22]. Moreover, there is evidence that knowledge, attitude and behaviours towards biological risk is an issue outside the health care sector [23-26].

Although several occupations entail biological risks, in Italy most of the data has been collected in hospital settings and, more specifically, by surveys of nurses [27, 28].

The aim of this study is to investigate knowledge and perception of occupational biological risk among workers in several occupations.

\section{METHODS}

\section{The questionnaire and setting}

The study was conducted by using a questionnaire with 33 items, divided in four main parts:

- socio-demographic data: gender, age, civil status, educational level, and type of work (public activity, industry, health and other);

- perception of the biological risks in ordinary occupational activity;

- knowledge about biological risks;

- biological risks in the working environment.

The questionnaire was submitted to a convenience sample of workers of an industrial area in Southern Rome, who were present at the working areas during the day of investigation with the consensus of the Company Director. The work fields that were investigated were the following: food, catering, service, farming and breeding, healthcare, school and research. Before the distribution of the questionnaires, the person in charge of administering these questionnaires presented the aims of the study, stressed the anonymity of the responders, and acquired informed written consent. The completed questionnaires were inserted in an secured urn.

The survey was carried out in the period of MarchApril 2010.

\section{The sample size calculations where:}

- total population size: 150000 workers in the selected fields (data from ISTAT);

- estimated expected frequency of knowledge on biological risks of the workers: this estimation was fixed at $50 \%$, considering a generic null hypothesis that half of workers have a good comprehensive knowledge on biological risk;

- worst acceptable of frequency of knowledge on biological risks in the work field $40 \%$.

Given the above parameters, and a confidence level of $99 \%$ we calculated a sample size of 661 individuals. Considering a $10 \%$ possible rate of non responders we decided to recruit at least 723 individuals.

The presentation of results was made according to the STROBE statement [29].

\section{Statistical analysis}

Data input was realized by using software Db-IV. The statistical analysis was performed using SPSS 19.00 for Windows.

Internal consistency was evaluated by using Cronbach's alpha on two set of items:

- the first one on three questions about perception of the biological risks in ordinary activity (see Table 1: questions number A1-A5);

- the second one on knowledge about the biological risks (see Table 1: questions number B1-B6).

Issues concerning safety instruments and procedures used on one's work site to reduce the biological risk were not analyzed as they depend from the particular activity that is carried out.

The descriptive statistics were carried out by using frequencies and percentages.

The analysis of possible associations between different groups of workers, knowledge and/or perception of the biological risk, was performed by using the Chi-square tests.

The level of significance was set at $\mathrm{p}<0.05$.

\section{RESULTS \\ Internal consistency}

The Cronbach's alpha computed for perception and knowledge of biological risk were 0.502 and 0.559 respectively (Table 1). 
Table 1 | Cronbach's alpha to evaluate the internal consistence on the perception and knowledge about biological risk, using questionnaire's main aggregate items

\begin{tabular}{|c|c|c|c|}
\hline \multicolumn{2}{|c|}{ Questions } & $\begin{array}{l}\text { Cronbach's } \\
\text { alpha if item } \\
\text { deleted }\end{array}$ & $\begin{array}{l}\text { Cronbach's } \\
\text { alpha of the } \\
\text { model }\end{array}$ \\
\hline \multicolumn{4}{|c|}{ Items concerning the biological risk perception } \\
\hline A1 & Are you really worried about biological risk? (Yes/No) & 0.366 & \multirow[t]{5}{*}{0.502} \\
\hline A2 & $\begin{array}{l}\text { In which ranking do you put the biological risk compared to chemical, physical, psychological } \\
\text { or management ones? }\left(1^{\text {th }}-4^{\text {th }}\right)\end{array}$ & 0.507 & \\
\hline A3 & Are personal events causes of this preoccupation? (Yes/No) & 0.483 & \\
\hline A4 & Is biological risk outstanding for you? (Yes/No) & 0.428 & \\
\hline A5 & Do you believe that your professional activity presents biological risk? (Yes/No) & 0.480 & \\
\hline \multicolumn{4}{|c|}{ Items concerning knowledge on biological risk } \\
\hline B1 & $\begin{array}{l}\text { Which of these common vehicles is to consider more infective? From } 1 \text { to } 9 \text { possible answers: vomit, } \\
\text { sexual activity, air, skin, urine, feces, blood, tears, sweat }\end{array}$ & 0.569 & \multirow[t]{6}{*}{0.559} \\
\hline B2 & $\begin{array}{l}\text { What do biological agents bring? From } 1 \text { to } 3 \text { possible answers: infectious diseases, intoxications, } \\
\text { allergies }\end{array}$ & 0.544 & \\
\hline B3 & Are the effects caused by biological agents sometimes treatable? (Yes/No) & 0.576 & \\
\hline B4 & $\begin{array}{l}\text { If the diseases are curable, how this could happen? (possible from } 1 \text { to } 4 \text { answers) spontaneous } \\
\text { recovery, relax, one cycle of treatment, more cycles of treatments }\end{array}$ & 0.546 & \\
\hline B5 & How do you assess your knowledge on the biological risk? (scale $1=$ scarce to $5=$ complete) & 0.462 & \\
\hline B6 & $\begin{array}{l}\text { Your knowledge on biological risk derives from: (possible from1 to } 5 \text { answers) research, } \\
\text { training and refresher courses, corporate training courses, congress or meeting, book or articles }\end{array}$ & 0.532 & \\
\hline
\end{tabular}

\section{Sample description}

Out of the 940 questionnaires that were distributed, $729(77.55 \%)$ were returned. The descriptive variables of the sample were reported in Table 2: males

\begin{tabular}{|c|c|c|c|}
\hline Variables & & no. & $\%$ \\
\hline \multirow[t]{3}{*}{ Gender } & Male & 416 & 57.20 \\
\hline & Female & 311 & 42.87 \\
\hline & Total of responders & 727 & 100.00 \\
\hline \multirow[t]{6}{*}{ Civil status } & Married & 443 & 61.70 \\
\hline & Widower & 7 & 0.97 \\
\hline & Divorced & 21 & 2.92 \\
\hline & Apart & 34 & 4.74 \\
\hline & Unmarried & 213 & 29.67 \\
\hline & Total of responders & 718 & 100.00 \\
\hline \multirow[t]{4}{*}{ Work area } & Food, catering & 147 & 20.16 \\
\hline & Service, farming and breeding & 249 & 34.16 \\
\hline & Health, school and research & 333 & 45.68 \\
\hline & Total of responders & 729 & 100.00 \\
\hline \multirow[t]{3}{*}{ Age (years) } & Mean & \multicolumn{2}{|c|}{37.37} \\
\hline & SD & \multicolumn{2}{|c|}{10.95} \\
\hline & Total of responders & \multicolumn{2}{|c|}{702} \\
\hline \multirow{3}{*}{$\begin{array}{l}\text { Years } \\
\text { working }\end{array}$} & Mean & \multicolumn{2}{|c|}{14.49} \\
\hline & SD & \multicolumn{2}{|c|}{10.57} \\
\hline & Total of responders & \multicolumn{2}{|c|}{678} \\
\hline
\end{tabular}

accounted for $57.2 \%$ of the total sample that had a mean age of 37.37 years $(\mathrm{SD}=10.95)$.

In Table 2 the distributions of socio-demographic information coming from the different work settings are revealed.

Table 3 shows the evaluation of the possible association between different workers versus biological risk perception items. Significant associations were found between different activity areas with respect to the relevance of the biological risk (question number A4) and the perception in our activities of the biological risk (question number A5), with $\mathrm{p}=0.044$ and $\mathrm{p}<0.001$ respectively.

Table 4 shows the univariate analysis evaluating the possible association between different workers and the knowledge of biological risk.

In particular, the percentages of the most common biological risk exposures (B1) were: air and physical contact for the catering and food group, $66.7 \%$ and $61.90 \%$ respectively; air and blood for the health and research group, with $73.50 \%$ and $57.00 \%$ respectively; and physical contact and blood for the service group, $63.10 \%$ and $48.30 \%$. Significantly different associations were found on the basis of the professional groups and the types of biological risk exposure after contact with: vomit $(\mathrm{p}<0.001)$, urine $(\mathrm{p}<0.001)$, feces $(<0.001)$ and physical contact $(p=0.002)$. All three groups reported that the prevalent effect caused by the biological agents (B2) was the occurrence of infectious diseases $(59.90 \%$ food group, $91.60 \%$ health and research and $79.30 \%$ service group), with a significant difference of pro- 


\begin{tabular}{|c|c|c|c|c|c|c|}
\hline \multirow{2}{*}{\multicolumn{2}{|c|}{ Questions about biological risk perception }} & \multicolumn{3}{|c|}{ Working areas } & \multirow{2}{*}{$\begin{array}{c}\text { Total } \\
\text { no. }\end{array}$} & \multirow[b]{2}{*}{$\mathbf{p}^{*}$} \\
\hline & & \multirow{2}{*}{$\begin{array}{c}\begin{array}{c}\text { Food or } \\
\text { catering }\end{array} \\
83 \\
22.93 \\
\end{array}$} & \multirow{2}{*}{$\begin{array}{c}\begin{array}{c}\text { Health or } \\
\text { research or } \\
\text { teaching }\end{array} \\
123 \\
33.98\end{array}$} & \multirow{2}{*}{$\begin{array}{c}\begin{array}{c}\text { Service, } \\
\text { farming or } \\
\text { breeding }\end{array} \\
156 \\
43.09\end{array}$} & & \\
\hline $\begin{array}{l}\text { A1. Are you really worried about } \\
\text { biological risk? }\end{array}$ & $\begin{array}{l}\text { no } \\
\%\end{array}$ & & & & $\begin{array}{c}362 \\
100.00\end{array}$ & \multirow[t]{3}{*}{0.096} \\
\hline & $\begin{array}{l}\text { yes } \\
\%\end{array}$ & $\begin{array}{c}49 \\
16.44\end{array}$ & $\begin{array}{c}103 \\
34.56\end{array}$ & $\begin{array}{c}146 \\
48.99\end{array}$ & $\begin{array}{c}298 \\
100.00\end{array}$ & \\
\hline & Total of responders & 132 & 226 & 302 & 660 & \\
\hline \multirow{5}{*}{$\begin{array}{l}\text { A2. In which ranking do you put } \\
\text { the biological risk compared to } \\
\text { chemical, physical, psychological } \\
\text { or management ones? }\end{array}$} & $\begin{array}{l}1^{\circ} \text { place } \\
\%\end{array}$ & $\begin{array}{c}15 \\
13.76\end{array}$ & $\begin{array}{c}42 \\
38.53\end{array}$ & $\begin{array}{c}52 \\
47.71\end{array}$ & $\begin{array}{c}109 \\
100.00\end{array}$ & \multirow[t]{5}{*}{0.370} \\
\hline & $\begin{array}{l}2^{\circ} \text { place } \\
\%\end{array}$ & $\begin{array}{c}24 \\
16.90\end{array}$ & $\begin{array}{c}49 \\
34.51\end{array}$ & $\begin{array}{c}69 \\
48.59\end{array}$ & $\begin{array}{c}142 \\
100.00\end{array}$ & \\
\hline & $\begin{array}{l}3^{\circ} \text { place } \\
\%\end{array}$ & $\begin{array}{c}29 \\
24.79\end{array}$ & $\begin{array}{c}40 \\
34.19\end{array}$ & $\begin{array}{c}48 \\
41.03\end{array}$ & $\begin{array}{c}117 \\
100.00\end{array}$ & \\
\hline & $\begin{array}{l}>3^{\circ} \text { place } \\
\%\end{array}$ & $\begin{array}{c}79 \\
21.88\end{array}$ & $\begin{array}{c}118 \\
32.69\end{array}$ & $\begin{array}{c}164 \\
45.43\end{array}$ & $\begin{array}{c}361 \\
100.00\end{array}$ & \\
\hline & Total of responders & 147 & 249 & 333 & 729 & \\
\hline \multirow[t]{3}{*}{$\begin{array}{l}\text { A3. Are personal events the causes } \\
\text { of this preoccupation? }\end{array}$} & $\begin{array}{l}\text { no } \\
\%\end{array}$ & $\begin{array}{c}81 \\
19.10\end{array}$ & $\begin{array}{c}151 \\
35.61\end{array}$ & $\begin{array}{c}192 \\
45.28\end{array}$ & $\begin{array}{c}424 \\
100.00\end{array}$ & \multirow[t]{3}{*}{0.147} \\
\hline & $\begin{array}{l}\text { yes } \\
\%\end{array}$ & $\begin{array}{c}7 \\
13.46\end{array}$ & $\begin{array}{c}14 \\
26.922\end{array}$ & $\begin{array}{c}31 \\
59.62\end{array}$ & $\begin{array}{c}52 \\
100.00\end{array}$ & \\
\hline & Total of responders & 88 & 165 & 223 & 476 & \\
\hline \multirow[t]{3}{*}{$\begin{array}{l}\text { A4. Is biological risk outstanding for } \\
\text { you? }\end{array}$} & $\begin{array}{l}\text { no } \\
\%\end{array}$ & $\begin{array}{c}20 \\
18.87\end{array}$ & $\begin{array}{c}27 \\
25.47\end{array}$ & $\begin{array}{c}59 \\
55.66\end{array}$ & $\begin{array}{c}106 \\
100.00\end{array}$ & \multirow[t]{3}{*}{0.044} \\
\hline & $\begin{array}{l}\text { yes } \\
\%\end{array}$ & $\begin{array}{c}93 \\
17.55\end{array}$ & $\begin{array}{c}201 \\
37.92\end{array}$ & $\begin{array}{c}236 \\
44.53\end{array}$ & $\begin{array}{c}530 \\
100.00\end{array}$ & \\
\hline & Total of responders & 113 & 228 & 295 & 636 & \\
\hline \multirow{4}{*}{$\begin{array}{l}\text { A5. Do you believe that your } \\
\text { professional activity presents } \\
\text { biological risk? }\end{array}$} & $\begin{array}{l}\text { no } \\
\%\end{array}$ & $\begin{array}{c}53 \\
36.55\end{array}$ & $\begin{array}{c}30 \\
20.69\end{array}$ & $\begin{array}{c}62 \\
42.76\end{array}$ & $\begin{array}{c}145 \\
100.00\end{array}$ & \multirow[t]{4}{*}{$<0.001$} \\
\hline & $\begin{array}{l}\text { yes } \\
\%\end{array}$ & $\begin{array}{c}88 \\
15.83\end{array}$ & $\begin{array}{c}215 \\
38.67\end{array}$ & $\begin{array}{c}253 \\
45.50\end{array}$ & $\begin{array}{c}556 \\
100.00\end{array}$ & \\
\hline & $\begin{array}{l}\text { I don't know } \\
\%\end{array}$ & $\begin{array}{c}6 \\
24.00\end{array}$ & $\begin{array}{c}1 \\
4.00\end{array}$ & $\begin{array}{c}18 \\
72.00\end{array}$ & $\begin{array}{c}25 \\
100.00\end{array}$ & \\
\hline & Total of responders & 147 & 246 & 333 & 726 & \\
\hline
\end{tabular}

portions that was found at $\mathrm{p}<0.001$. Regarding intoxications and allergies, significant differences percentage were also computed, respectively at $\mathrm{p}<$ 0.001 and $\mathrm{p}=0.002$.

These results were probably related to the educational levels and the individual awareness about pollution of the specific materials (i.e. solid waste, waste water, slaughtered materials, blood etc.).

Although food chain workers are involved in the educational programs about hazard and risk, we found that, surprisingly, they are less aware of the topic compared to health and service workers.

For question number B4, investigating the types of recoveries, the option "with more cycles of treatments" was more indicated from all groups: $62.2 \%$ food group, $58.60 \%$ health and research group and $57.10 \%$ service group.

The perception of own knowledge (B5) resulted in a good rank (sufficient, many or complete) in the food and catering group, $78.3 \%$ with significant difference compared to other professions $(\mathrm{p}<0.001)$. The sources of knowledge were significantly different (B6), with $62.60 \%$ of the food group and $48.90 \%$ of the service group declaring to have received major support from corporate training courses, while $47.80 \%$ of the health and research group received support from scientific studies.

\section{DISCUSSION}

Our results suggest that the study was able to adequately test knowledge on worker's biological risk but not perception of risk (Cronbach's alpha 0.559 vs 0.502). But the low level of internal consistency could be considered acceptable if the "underlying construct" is multifaceted [30], such as in this case. 


\begin{tabular}{|c|c|c|c|c|c|c|c|c|c|c|}
\hline \multirow{2}{*}{\multicolumn{3}{|c|}{ Questions concerning biological risk knowledge }} & \multicolumn{6}{|c|}{ Working areas } & \multirow{3}{*}{$\begin{array}{c}\text { Total } \\
\text { no. } \\
672 \\
\end{array}$} & \multirow[b]{2}{*}{$\mathbf{p}^{\star}$} \\
\hline & & & \multicolumn{2}{|c|}{$\begin{array}{l}\text { Food } \\
\text { or Catering }\end{array}$} & \multicolumn{2}{|c|}{$\begin{array}{l}\text { Health research } \\
\text { or teaching }\end{array}$} & \multicolumn{2}{|c|}{$\begin{array}{l}\text { Service farming } \\
\text { or breeding }\end{array}$} & & \\
\hline \multirow{27}{*}{$\begin{array}{l}\text { B1. Which of these } \\
\text { common vehicles } \\
\text { is to consider } \\
\text { more infective? }\end{array}$} & \multirow[t]{3}{*}{ Vomit } & No (\%) & 141 & $(95.9)$ & 212 & $(85.1)$ & 319 & $(95.8)$ & & $<0.001$ \\
\hline & & Yes (\%) & 6 & (4.1) & 37 & (14.9) & 14 & (4.2) & 57 & \\
\hline & & Total & 147 & & 249 & & 333 & & 729 & \\
\hline & \multirow{3}{*}{$\begin{array}{l}\text { Sexual } \\
\text { relations }\end{array}$} & № $(\%)$ & 94 & $(63.90)$ & 172 & $(69.10)$ & 240 & (72.1) & 506 & 0.203 \\
\hline & & Yes $(\%)$ & 53 & $(36.10)$ & 77 & $(30.90)$ & 93 & (27.9) & 223 & \\
\hline & & Total & 147 & & 249 & & 333 & & 729 & \\
\hline & \multirow[t]{3}{*}{ Air } & No (\%) & 49 & $(33.30)$ & 66 & $(26.50)$ & 117 & $(35.10)$ & 232 & 0.079 \\
\hline & & Yes (\%) & 98 & $(66.7)$ & 183 & $(73.50)$ & 216 & $(43.46)$ & 497 & \\
\hline & & Total & 147 & & 249 & & 333 & & 729 & \\
\hline & \multirow{3}{*}{$\begin{array}{l}\text { Physical } \\
\text { contact }\end{array}$} & No (\%) & 56 & $(38.10)$ & 126 & $(50.60)$ & 123 & $(36.90)$ & 305 & 0.002 \\
\hline & & Yes (\%) & 91 & $(61.90)$ & 123 & $(49.40)$ & 210 & $(63.10)$ & 424 & \\
\hline & & Total & 147 & & 249 & & 333 & & 729 & \\
\hline & \multirow[t]{3}{*}{ Urine } & № (\%) & 129 & $(87.80)$ & 190 & $(76.30)$ & 294 & (88.30) & 613 & $<0.001$ \\
\hline & & Yes (\%) & 18 & (12.20) & 59 & $(23.70)$ & 39 & (11.70) & 116 & \\
\hline & & Total & 147 & & 249 & & 333 & & 729 & \\
\hline & \multirow[t]{3}{*}{ Feces } & No (\%) & 121 & $(82.30)$ & 167 & $(67.10)$ & 279 & $(83.80)$ & 567 & $<0.001$ \\
\hline & & Yes (\%) & 26 & (17.70) & 82 & $(32.90)$ & 54 & (16.20) & 162 & \\
\hline & & Total & 147 & & 249 & & 333 & & 729 & \\
\hline & \multirow[t]{3}{*}{ Blood } & № (\%) & 69 & $(46.90)$ & 107 & $(43.00)$ & 172 & $(51.70)$ & 348 & 0.114 \\
\hline & & Yes (\%) & 78 & (53.10) & 142 & $(57.00)$ & 161 & (48.30) & 381 & \\
\hline & & Total & 147 & & 249 & & 333 & & 729 & \\
\hline & \multirow[t]{3}{*}{ Tears } & No (\%) & 147 & (100.00) & 242 & $(97.20)$ & 328 & $(98.50)$ & 717 & n.c. \\
\hline & & Yes (\%) & 0 & $(0.00)$ & 7 & $(2.80)$ & 5 & (1.50) & 12 & \\
\hline & & Total & 147 & & 249 & & 333 & & 729 & \\
\hline & \multirow[t]{3}{*}{ Sweat } & No (\%) & 143 & $(97.30)$ & 244 & $(98.00)$ & 325 & $(97.60)$ & 712 & 0.896 \\
\hline & & Yes (\%) & 4 & $(2.70)$ & 5 & (2.00) & 8 & (2.40) & 17 & \\
\hline & & Total & 147 & & 249 & & 333 & & 729 & \\
\hline \multirow{9}{*}{$\begin{array}{l}\text { B2. What do biological } \\
\text { agents bring? }\end{array}$} & \multirow{3}{*}{$\begin{array}{l}\text { Infectious } \\
\text { disease }\end{array}$} & № (\%) & 59 & $(40.10)$ & 21 & $(8.40)$ & 69 & $(20.70)$ & 149 & $<0.001$ \\
\hline & & Yes (\%) & 88 & $(59.90)$ & 228 & $(91.60)$ & 264 & $(79.30)$ & 580 & \\
\hline & & Total (\%) & 147 & & 249 & & 333 & & 729 & \\
\hline & \multirow[t]{3}{*}{ Intoxications } & № (\%) & 72 & $(49.00)$ & 160 & $(64.30)$ & 235 & $(70.60)$ & 467 & $<0.001$ \\
\hline & & Yes (\%) & 75 & $(51.00)$ & 89 & $(35.70)$ & 98 & $(29.40)$ & 262 & \\
\hline & & Total & 147 & & 249 & & 333 & & 729 & \\
\hline & Allergies & No (\%) & 89 & $(60.50)$ & 159 & $(63.90)$ & 166 & $(49.80)$ & 414 & 0.002 \\
\hline & & Yes $(\%)$ & 58 & $(39.50)$ & 90 & $(36.10)$ & 167 & $(50.20)$ & 315 & \\
\hline & & Total & 147 & & 249 & & 333 & & 729 & \\
\hline B3. Are the effects/dise & & № (\%) & 29 & $(20.30)$ & 33 & $(13.40)$ & 34 & $(10.60)$ & 96 & 0.018 \\
\hline caused by biological & & Yes (\%) & 114 & $(79.70)$ & 214 & $(86.60)$ & 288 & $(89.40)$ & 616 & \\
\hline sometimes treatabl & & Total & 147 & & 249 & & 333 & & 712 & \\
\hline B4. If the diseases are & Spontaneous & № (\%) & 143 & (97.3) & 239 & $(96.00)$ & 325 & $(97.60)$ & 707 & $<0.001$ \\
\hline curable, how this & recovery & Yes (\%) & 4 & $(2.70)$ & 10 & $(4.00)$ & 8 & (2.40) & 22 & \\
\hline & & Total & 147 & & 249 & & 333 & & 729 & \\
\hline & Relax & № (\%) & 143 & $(97.30)$ & 246 & $(98.80)$ & 329 & $(98.80)$ & 718 & 0.402 \\
\hline & & Yes (\%) & 4 & $(2.70)$ & 3 & $(1.20)$ & 4 & (1.20) & 11 & \\
\hline & & Total & 147 & & 249 & & 333 & & 729 & \\
\hline
\end{tabular}




\begin{tabular}{|c|c|c|c|c|c|c|c|c|c|c|}
\hline \multirow{2}{*}{\multicolumn{3}{|c|}{ Questions concerning biological risk knowledge }} & \multicolumn{6}{|c|}{ Working areas } & \multirow[b]{2}{*}{$\begin{array}{c}\text { Total } \\
\text { no. }\end{array}$} & \multirow[b]{2}{*}{$\mathbf{p}^{\star}$} \\
\hline & & & \multicolumn{2}{|c|}{$\begin{array}{l}\text { Food or } \\
\text { Catering }\end{array}$} & \multicolumn{2}{|c|}{$\begin{array}{l}\text { Health or } \\
\text { research } \\
\text { or teaching }\end{array}$} & \multicolumn{2}{|c|}{$\begin{array}{l}\text { Service or } \\
\text { farming } \\
\text { or breeding }\end{array}$} & & \\
\hline \multirow{6}{*}{$\begin{array}{l}\text { B4. If the diseases are } \\
\text { curable, how this } \\
\text { could happen? }\end{array}$} & \multirow{3}{*}{$\begin{array}{l}\text { One cycle of } \\
\text { treatment }\end{array}$} & No (\%) & 104 & $(70.70)$ & 146 & $(58.60)$ & 208 & $(62.50)$ & 458 & \multirow{3}{*}{0.054} \\
\hline & & Yes $(\%)$ & 43 & $(29.30)$ & 103 & $(41.40)$ & 125 & $(37.50)$ & 271 & \\
\hline & & Total & 147 & & 249 & & 333 & & 729 & \\
\hline & \multirow{3}{*}{$\begin{array}{l}\text { More cycles } \\
\text { of treatments }\end{array}$} & № (\%) & 55 & $(37.4)$ & 103 & $(41.40)$ & 143 & $(42.90)$ & 301 & \multirow[t]{3}{*}{0.527} \\
\hline & & Yes (\%) & 92 & $(62.2)$ & 146 & $(58.60)$ & 190 & $(57.10)$ & 428 & \\
\hline & & Total & 147 & & 249 & & 333 & & 729 & \\
\hline \multirow{6}{*}{\multicolumn{2}{|c|}{$\begin{array}{l}\text { B5. How do you assess } \\
\text { your knowledge } \\
\text { on the biological risk? }\end{array}$}} & scarce $(\%)$ & 4 & $(2.80)$ & 2 & $(0.80)$ & 10 & $(3.00)$ & 16 & \multirow[t]{6}{*}{0.019} \\
\hline & & Insufficient (\%) & 27 & $(18.90)$ & 53 & $(21.80)$ & 72 & $(22.00)$ & 152 & \\
\hline & & Sufficient (\%) & 89 & $(62.20)$ & 116 & $(47.70)$ & 185 & $(56.40)$ & 390 & \\
\hline & & Good (\%) & 19 & $(13.30)$ & 57 & $(23.50)$ & 49 & $(14.90)$ & 125 & \\
\hline & & Complete (\%) & 4 & $(2.80)$ & 15 & $(6.20)$ & 12 & $(3.70)$ & 31 & \\
\hline & & Total & 143 & & 243 & & 328 & & 714 & \\
\hline \multirow{15}{*}{$\begin{array}{l}\text { B6. Your knowledge } \\
\text { on biological risk } \\
\text { derives from? }\end{array}$} & \multirow[t]{3}{*}{ Research } & No $(\%)$ & 139 & $(94.60)$ & 130 & $(52.20)$ & 286 & $(85.90)$ & 555 & \multirow{3}{*}{$<0.001$} \\
\hline & & Yes $(\%)$ & 8 & $(5.40)$ & 119 & $(47.80)$ & 47 & (14.10) & 174 & \\
\hline & & Total & 147 & & 249 & & 333 & & 729 & \\
\hline & \multirow{3}{*}{$\begin{array}{l}\text { Training and } \\
\text { refresher } \\
\text { courses }\end{array}$} & № (\%) & 138 & $(93.90)$ & 216 & $(86.70)$ & 315 & $(94.60)$ & 669 & \multirow[t]{3}{*}{0.002} \\
\hline & & Yes (\%) & 9 & $(6.10)$ & 33 & $(13.30)$ & 18 & $(5.40)$ & 60 & \\
\hline & & Total & 147 & & 249 & & 333 & & 729 & \\
\hline & \multirow{3}{*}{$\begin{array}{l}\text { Corporate } \\
\text { training } \\
\text { courses }\end{array}$} & No (\%) & 55 & $(37.40)$ & 174 & $(69.90)$ & 170 & $(51.10)$ & 399 & \multirow{3}{*}{$<0.001$} \\
\hline & & Yes $(\%)$ & 92 & $(62.60)$ & 75 & $(30.10)$ & 163 & $(48.90)$ & 330 & \\
\hline & & Total & 147 & & 249 & & 333 & & 729 & \\
\hline & \multirow{3}{*}{$\begin{array}{l}\text { Congress/ } \\
\text { meeting }\end{array}$} & No $(\%)$ & 144 & $(98.00)$ & 218 & $(87.60)$ & 316 & $(94.90)$ & 678 & \multirow{3}{*}{$<0.001$} \\
\hline & & Yes $(\%)$ & 3 & $(2.00)$ & 31 & (12.40) & 17 & $(5.10)$ & 51 & \\
\hline & & Total & 147 & & 249 & & 333 & & 729 & \\
\hline & \multirow{3}{*}{$\begin{array}{l}\text { Books or } \\
\text { articles }\end{array}$} & No (\%) & 106 & (72.10) & 144 & $(57.80)$ & 202 & $(60.70)$ & 452 & \multirow[t]{3}{*}{0.015} \\
\hline & & Yes (\%) & 41 & $(27.90)$ & 105 & $(42.20)$ & 131 & $(39.30)$ & 277 & \\
\hline & & Total & 147 & & 249 & & 333 & & 729 & \\
\hline
\end{tabular}

The univariate analysis for the evaluation of the possible association between workers' status and biological risk perception shows a significant relationship between the answers to the importance of the biological risk and the biological risk exposure in our activities ( $\mathrm{p}=0.044$ and $\mathrm{p}<0.001$, respectively).

Data reveals a high level of preoccupations for biological risk $(48.34 \%)$ in the working areas of service, farming and breeding, confirming the results that other authors reported in their studies $[6,8]$. Farmers are at high risk for exposure to airborne dust and microorganisms which can be significant during harvesting season.

Almost half of the workers (49.5\%) rank biological risk third and 15\% rank biological risk first amongst occupational risks. These data are in agreement with the lack of unfavorable experiences, as evidenced by $89.1 \%$ of responders.
Our study pinpointed an acknowledgment of the role of air and blood transmission, mostly considered by health care workers and researchers or teachers $(73.5 \%$ and $57.1 \%$ respectively); while the role of tears and sweat was poorly recognized by all participants. In particular, our data confirm the recognition of health professionals as the most exposed workers both for contact with blood and body fluids and high risk of accidental occupational exposure [31]. Retrospective studies indicate a cumulative prevalence of almost $50 \%$ of work injuries, with high prevalence of needle puncture, particularly on hands $(38,58)$. Infectious diseases sustained by $B$. tuberculosis, tularemia, Streptococcus A and Staphylococcus aureus are occupational risks for healthcare workers $[9,32,33]$.

All participants show good knowledge of effects induced by biological agents and it is significant that almost half of the respondents are aware of the risks 
concerning allergies. Nevertheless, it is surprising that this risk is known mainly by workers of service, farming and breeding, to a greater extent than it is known to health workers. In that case, the study suggests the need of specific training on biological risk in order to reduce lack of knowledge in this area.

Most workers $(86 \%)$ consider there is a good chance that workers who get an infectious disease at work will recover, mainly with more cycles of treatments.

Moreover, a significant association was found in the answers concerning the sources of knowledge. It is surprising that less than half of responders reported corporate training courses $(45.3 \%)$ as the main sources of knowledge on the biological risk among workers, since this type of information is mandatory according to actual health and safety legislation. This low percentage could be either due to lack of intervention or lack of quality in this field. Moreover, books or articles, as well as congress or meetings, have been considered by $38 \%$ and $7 \%$ of responders, respectively.

The data altogether has pointed out differences of biological risk perception or its knowledge in the various working areas. The participants show a perception of own occupational biological risk aligned with their knowledge. This concerns workers of service, and farming and breeding workers, who have not only pointed out greater preoccupation about biological risk (48.3\%) than other workers, but also greater knowledge regarding risks, especially allergies $(50.1 \%)$.

The EU has restricted the risk of occupational exposure to biological agents with the Directive issued on the $18^{\text {th }}$ of September 2000 [34], with numerous institutions currently taking care of biological risks. Within the European Community strategy 20022006 [35], the European Risk Observatory of the European Agency for Safety and Health at Work identified the ten most important emerging biological risks [36]: occupational risks related to global epidemics, difficult assessment of biological risks, workers exposure to drug-resistant microorganisms, lack of information on biological risks, poor maintenance of air-conditioning and water systems, inadequate $\mathrm{OSH}$ (Occupational Safety Health) training of local authorities staff, biohazards in waste treatment plants, combined exposure to bioaerosols and chemicals, endotoxins, and moulds in indoor workplaces.

Finally, we need to consider the difficulty of biological risk assessment. Limit values of the presence of the biological agent have not yet been established and this prevents the implementation of a revised risk and effects assessment. The influence of the effects of genetic susceptibility to infectious diseases also needs to be considered [37]. Continued surveillance for cases of biological diseases is necessary in order to determine incidence, as well as specific sources and procedures representing risk factors.

In conclusion, this study underlined the importance of the biological risk not only in the healthcare sector, but also in other work fields, such as food, catering, service, farming and breeding, school and research. However, even if almost half of participants have the perception of the importance of biological risk, knowledge on this issue is still lacking, and this suggests the need of specific training on biological risk in order to reduce lack of knowledge in this area.

\section{Conflict of interest statement}

There are no potential conflicts of interest or any financial or personal relationships with other people or organizations that could inappropriately bias conduct and findings of this study.

Received on 12 September 2011.

Accepted on 4 January 2012.

\section{References}

1. Driscoll T, Takala J, Steenland K, Corvalan C, Fingerhut M. Review of estimates of the global burden of injury and illness due to occupational exposures. Am J Ind Med 2005; 48(6):491-502. http://dx.doi.org/10.1002/ajim.20194

2. Castiglia P, Liguori G, Montagna MT, Napoli C, Pasquarella C, Bergomi M, Fabiani L, Monarca S, Petti S; SItI Working Group Hygiene in Dentistry. Italian multicenter study on infection hazards during dental practice: control of environmental microbial contamination in public dental surgeries. BMC Public Health 2008;8:187.

http://dx.doi.org/10.1186/1471-2458-8-187

3. Corrao CRN, Biagi M, Tantardini M. Accident risk evaluation in dentistry. Proceeding XII Congress of the International Commission on Occupational Health (ICOH) Scientific Committee "Towards a multidimensional approach in occupational health service: scientific evidence, social consensus, human values" (Modena 13-16 october 2004). Modena: ICOH Scientific Committee; 2004. p. 18.

4. Vaquero M, Gómez P, Romero M, Casal MJ, Spanish Group of Mycobacteriology. Investigation of biological risk in my-

cobacteriology laboratories: a multicentre study. Int J Tuberc Lung Dis 2003;7(9):879-85.

5. Mansi A, Paba E, Marcelloni AM, Bruni R, Spagnoli G. Legionnaires' disease and occupational risk: a case report. Ital J Public Health 2004;87-8.

6. Dutkiewicz J, Krysin'ska-Traczyk E, Skórska C, Cholewa G, Sitkowska J. Exposure to airborne microorganisms and endotoxin in a potato processing plant. Ann Agric Environ Med 2002;9(2):225-35.

7. Oguttu JW, Veary CM, Picard JA. Antimicrobial drug resistance of Escherichia coli isolated from poultry abattoir workers at risk and broilers on antimicrobials. J S Afr Vet Assoc 2008; 79(4):161-6.

8. Schierl R, Heise A, Egger U, Schneider F, Eichelser R, Neser S, Nowak D. Endotoxin concentration in modern animal houses in southern Bavaria. Ann Agric Environ Med 2007;14(1):129-36.

9. Alvarado-Esquivel C, Liesenfeld O, Márquez-Conde JA, Cisneros-Camacho A, Estrada-Martínez S, Martínez-García 
SA, González-Herrera A, García-Corral N. Seroepidemiology of infection with Toxoplasma gondii in waste pickers and waste workers in Durango, Mexico. Zoonoses Public Health 2008;55(6):306-12 http://dx.doi.org/10.1111/j.1863-2378.2008.01133.x

10. Dounias G, Kypraiou E, Rachiotis G, Tsovili E, Kostopoulos S. Prevalence of hepatitis B virus markers in municipal solid waste workers in Keratsini (Greece). Occup Med (Lond) 2005; 55(1):60-3. http://dx.doi.org/10.1093/occmed/kqi007

11. Grisoli P, Rodolfi M, Villani S, Grignani E, Cottica D, Berri A, Picco AM, Dacarro C. Assessment of airborne microorganism contamination in an industrial area characterized by an open composting facility and a wastewater treatment plant. Environ Res 2009;109(2):135-42. http://dx.doi.org/10.1016/j.envres.2008.11.001

12. Nadal M, Inza I, Schuhmacher M, Figueras MJ, Domingo JL. Health risks of the occupational exposure to microbiological and chemical pollutants in a municipal waste organic fraction treatment plant. Int J Hyg Environ Health 2009; 212(6):661-9. http://dx.doi.org/10.1016/j.ijheh.2009.06.002

13. Corrao CRN, Sabatini G, Farulla A. Occupational exposure to HIV in prison. Proceeding LV National Conference SIMLII (Turin 30 september-2 October 1992). Turin: Pas Information Publisher; 1992. p. 1805-8.

14. Dull PM, Wilson KE, Kournikakis B, Whitney EA, Boulet CA, Ho JY, Ogston J, Spence MR, McKenzie MM, Phelan MA, Popovic T, Ashford D. Bacillus anthracis aerosolization associated with a contaminated mail sorting machine. Emerg Infect Dis 2002;8(10):1044-7.

http://dx.doi.org/10.3201/eid0810.020356

15. Lowy FD, Aiello AE, Bhat M, Johnson-Lawrence VD, Lee MH, Burrell E, Wright LN, Vasquez G, Larson EL. Staphylococcus aureus colonization and infection in New York State prisons. J Infect Dis 2007;196(6):911-8. http://dx.doi.org/10.1086/520933

16. Madsen AM, Kruse P, Schneider T. Characterization of microbial particle release from biomass and building material surfaces for inhalation exposure risk assessment. Ann Occup Hyg 2006;50(2):175-87. http://dx.doi.org/10.1093/annhyg/mei057

17. Reiman M, Uitti J. Exposure to microbes, endotoxins and total dust in cigarette and cigar manufacturing: an evaluation of health hazards. Ann Occup Hyg 2000;44(6):467-73. http://dx.doi.org/10.1016/S0003-4878(00)00012-0

18. Pruss-Ustun A, Rapiti E, Hutin Y. Sharp injuries: global burden of disease from sharp injuries to health-care workers. WHO Environmental Burden of Disease Series, n. 3. Geneva: WHO; 2003.

19. Sreedharan J, Muttappallymyalil J, Venkatramana M. Knowledge and practice of standard measures in occupational exposure to blood and body fluids among nurses in a University Hospital in the United Arab Emirates. Ital J Public Health 2010;7(1):90-4.

20. Benítez Rodríguez E, Ruiz Moruno AJ, Córdoba Doña JA, Escolar Pujolar A, López Fernández FJ. Underreporting of percutaneous exposure accidents in a teaching hospital in Spain. Clin Perform Qual Health Care 1999;7(2):88-91.

21. Boal WL, Leiss JK, Sousa S, Lyden JT, Li J, Jagger J. The national study to prevent blood exposure in paramedics: exposure reporting. Am J Ind Med 2008;51(3):213-22. http://dx.doi.org/10.1002/ajim.20558

22. Vázquez-Mayoral EE, Sánchez-Pérez L, Olguín-Barreto Y, Acosta-Gío AE. Dental school deans' and dentists' perceptions of infection control and HIVIAIDS patient care: a challenge for dental education in Mexico. AIDS Patient Care STDS 2009;23(7):557-62. http://dx.doi.org/10.1089/apc.2008.0117

23. Candura SM, Verni P, Minelli CM, Rosso GL, Cappelli MI, Strambi S, Martellosio V. Rischi professionali nelle Forze dell'Ordine. G Ital Med Lav Ergon 2006;28(1):53-62.

24. Wang X, Wu S, Song Q, Tse LA, Yu IT, Wong TW, Griffiths S. Occupational health and safety challenges in China--focusing on township-village enterprises. Arch Environ Occup Health 2011;66(1):3-11. http://dx.doi.org/10.1080/19338244.2010.486424

25. Kozajda A, Szadkowska-Stan'czyk I. Activities of the National Information Center for Biological Agents present at workplace and preliminary analysis of data collected by the National Register of Biological Agents Med Pr 2009;60(4):327-34.

26. Rodríguez M, Valero A, Posada-Izquierdo GD, Carrasco E, Zurera G. Evaluation of food handler practices and microbiological status of ready-to-eat foods in long-term care facilities in the Andalusia region of Spain. J Food Prot 2011;74(9):1504-12. http://dx.doi.org/10.4315/0362-028X.JFP-10-468

27. Sindoni L, Calisto ML, Alfino D, Cannavò G, Grillo CO, Squeri R, Spagnolo EV. Retrospective survey on epidemiologic monitoring of accidents due to professional exposure to biological agents in A.O.U. "G. Martino" of Messina, Italy. Ann Ig 2005;17(1):67-74.

28. Daglio M, Sacchi M, Feletti T, Lanave M, Marena C, Zambianchi L, Strosselli M. Accidents due to biological risk in health personnel: descriptive epidemiological analysis of the decade 1994-2003. G Ital Med Lav Ergon 2006;28(4):457-65.

29. Little J, Higgins JPT, Ioannidis JPA, Moher D, Gagnon F, von Elm E, et al. Strengthening the REporting of Genetic Association Studies (STREGA). An Extension of the STROBE Statement. Ital J Public Health 2009;6(3):238-55. http://dx.doi.org/10.1111/j.1365-2362.2009.02125.x

30. Cronbach L J. Coefficient alpha and the internal structure of tests. Psychometrika 1951;16:297-334. http://dx.doi.org/10.1007/BF02310555

31. Lipscomb J, Sokas R, McPhaul K, Scharf B, Barker P, Trinkoff A, Storr C. Occupational blood exposure among unlicensed home care workers and home care registered nurses: are they protected? Am J Ind Med 2009;52(7):563-70. http://dx.doi.org/10.1002/ajim.20701

32. Lacy MD, Horn K. Nosocomial transmission of invasive group a streptococcus from patient to health care worker. Clin Infect Dis 2009;49(3):354-7. http://dx.doi.org/10.1086/599832

33. Shiomori T, Miyamoto H, Makishima K, Yoshida M, Fujiyoshi T, Udaka T, Inaba T, Hiraki N. Evaluation of bedmaking-related airborne and surface methicillin-resistant Staphylococcus aureus contamination. J Hosp Infect 2002; 50(1):30-5. http://dx.doi.org/10.1053/jhin.2001.1136

34. European Parliament. Directive 2000/54/EC on the protection of workers from risks related to exposure to biological agents at work. Official Journal L 262/21 of 17/10/2000.

35. European Union - Adapting to change in work and society: a new Community strategy on health and safety at work 2002-06 $-\operatorname{COM}(2002) 118$ final.

36. European Union. Expert forecast on emerging biological risks related to occupational safety and health. Available from: http://riskobservatory.osha.europa.eu/risks/forecasts/biological_risks.

37. Pietrangeli B. Biological risks in workplaces: research priorities for risk assessment. Prevention Today 2008;4(1):57-68. 